\title{
छs \\ Zero-temperature Monte Carlo study of the noncoplanar phase of the classical bilinear-biquadratic Heisenberg model on the triangular lattice
}

\author{
Sandro Wenzel, ${ }^{1}$ Sergey E. Korshunov, ${ }^{2}$ Karlo Penc, ${ }^{3}$ and Frédéric Mila ${ }^{1}$ \\ ${ }^{1}$ Institute of Theoretical Physics, Ecole Polytechnique Fédérale de Lausanne, CH-1015 Lausanne, Switzerland \\ ${ }^{2}$ L.D. Landau Institute for Theoretical Physics, Russian Academy of Sciences, 142432 Chernogolovka, Russia \\ ${ }^{3}$ Institute for Solid State Physics and Optics, Wigner Research Centre for Physics, Hungarian Academy of Sciences, \\ P.O.Box 49, H-1525 Budapest, Hungary
}

(Received 28 May 2013; published 4 September 2013)

\begin{abstract}
We investigate the ground-state properties of the highly degenerate noncoplanar phase of the classical bilinearbiquadratic Heisenberg model on the triangular lattice with Monte Carlo simulations. For that purpose, we introduce an Ising pseudospin representation of the ground states, and we use a simple Metropolis algorithm with local updates, as well as a powerful cluster algorithm. At sizes that can be sampled with local updates, the presence of long-range order is surprisingly combined with an algebraic decay of correlations and the complete disordering of the chirality. It is only thanks to the investigation of unusually large systems (containing $\sim 10^{8}$ spins) with cluster updates that the true asymptotic regime can be reached and that the system can be proven to consist of equivalent (i.e., equally ordered) sublattices. These large-scale simulations also demonstrate that the scalar chirality exhibits long-range order at zero temperature, implying that the system has to undergo a finite-temperature phase transition. Finally, we show that the average distance in the order parameter space, which has the structure of an infinite Cayley tree, remains remarkably small between any pair of points, even in the limit when the real space distance between them tends to infinity.
\end{abstract}

DOI: 10.1103/PhysRevB.88.094404

PACS number(s): 75.10.Hk, 75.50.Ee, 05.10.Ln

\section{INTRODUCTION}

It is hardly possible to overestimate the role played by the Heisenberg model with interactions of the type $\mathbf{S}_{i} \cdot \mathbf{S}_{j}$ between nearest neighbors in magnetism and more generally in statistical physics. However, when dealing with quantum spins $S$ larger than $1 / 2$, the most general rotationally invariant interaction is actually a polynomial of degree $2 S$ in $\mathbf{S}_{i} \cdot \mathbf{S}_{j}$. These models play a very important role in one dimension because, for specific polynomials, exact results have been derived, either because the model is integrable, ${ }^{1-3}$ or because the ground state can be constructed explicitly. ${ }^{4}$ For spin 1 , the corresponding Hamiltonian is the bilinear-biquadratic Heisenberg model defined by

$$
\mathcal{H}=J_{1} \sum_{(i j)} \mathbf{S}_{i} \cdot \mathbf{S}_{j}+J_{2} \sum_{(i j)}\left(\mathbf{S}_{i} \cdot \mathbf{S}_{j}\right)^{2} .
$$

The coupling constants $J_{1}$ and $J_{2}$ are conveniently parametrized by $J_{1}=J \cos \theta$ and $J_{2}=J \sin \theta$ with $\theta \in[-\pi, \pi)$.

A lot of attention has been focused recently on studying the same model on the triangular lattice, following the observation that the compound $\mathrm{NiGaS}_{4}$ does not possess any trace of standard periodic magnetic ordering at low temperatures. ${ }^{5}$ Accordingly, it has been suggested that the ground state is either a completely disordered spin-liquid or a spin-nematic phase. This suggestion has been supported by several theoretical investigations ${ }^{6-9}$ which have demonstrated that the spin-1 bilinear-biquadratic Heisenberg model (1) on the triangular lattice can indeed stabilize ferro- or antiferroquadrupolar order. Similar results have been obtained on the square lattice. ${ }^{10}$

It has been shown by Kawamura and Yamamoto ${ }^{11}$ that in the case of the triangular lattice the model defined by Eq. (1) has a rich phase diagram with various competing phases even in the classical limit when spins $\mathbf{S}$ are classical vectors of unit length.
After analyzing which spin configurations minimize the energy of a single triangular plaquette for different values of the parameter $\theta$, these authors have proposed a zero-temperature phase diagram with the notable presence of four different phases: a ferromagnetic phase (I), a spin-nematic phase (II), a three-sublattice antiferromagnetic $\left(120^{\circ}\right)$ phase (III), and a noncoplanar phase (IV).

While phases I-III are rather well understood on the basis of the results of Ref. 11 (which includes also numerical simulations), a thorough discussion of the noncoplanar phase IV was only recently given in Ref. 12 by three of the present authors. In that paper, the family of possible ground states has been systematically analyzed, and it has been shown that they can be represented in terms of domain wall configurations, giving rise to a loop model. It was further shown that the noncoplanar phase has an extensive ground-state degeneracy, which makes the question of whether at zero temperature the system is magnetically ordered, critical, or disordered a nontrivial one. By constructing a classification of the noncoplanar ground states in terms of an infinite Cayley tree of degree 3 , and by developing an analogy to an $O(3)$ model on a honeycomb lattice that has the same ground-state manifold, the authors of Ref. 12 have argued that these states are, except for two special values of the parameter $\theta$, magnetic states with a true long-range order in terms of spin orientation.

The main goal of this paper is to critically address this claim with an unbiased numerical approach based on a Monte Carlo (MC) method capable of efficiently sampling noncoplanar ground-state configurations. Another important motivation is to investigate whether the noncoplanar ground states are of chiral nature, since this question is beyond the scope of the analysis of Refs. 11 and 12. As we shall see, this task turns out to be rather involved, with the need to go to very large systems with roughly $10^{8}$ spins in order to reach a reliable conclusion. 
The paper is organized as follows. Section II reviews the classification of noncoplanar ground states of the bilinearbiquadratic model on the triangular lattice in terms of the zero-energy domain walls introduced in Ref. 12. In this section, we also show that these ground states can be mapped onto the states of an Ising-like (pseudospin) model defined on the same triangular lattice, which facilitates the numerical analysis of the family of ground states. Section III presents Monte Carlo results obtained for this Ising-like model using a local update technique on medium system sizes. While those numerical results are already in favor of magnetic ordering, they do not allow one to understand the precise nature of ordering and suggest the necessity to go to much larger length scales for clear conclusions. In Sec. IV, we introduce a nonlocal update mechanism capable of efficiently sampling the ground-state manifold up to very large system sizes and allowing to draw unambiguous conclusions regarding the nature of the phase. Section $\mathrm{V}$ is devoted to a presentation of those numerical results, and in Sec. VI, the conclusions and a summary are given.

\section{GROUND-STATE MANIFOLD AND THE PSEUDOSPIN REPRESENTATION}

\section{A. Ground-state manifold}

In the classical model defined by Hamiltonian (1), the energy of the interaction of two neighboring spins is given by

$$
E(\Phi)=J_{1} \cos \Phi+J_{2} \cos ^{2} \Phi,
$$

where $\Phi$ is the angle between them. For $\left|J_{1}\right| \leqslant 2 J_{2}$, this energy is minimized when $\Phi=\arccos \left(-J_{1} / 2 J_{2}\right)$. Accordingly, in the window $-2 J_{2}<J_{1}<J_{2}$, the angle $\Phi$ belongs to the interval $0<\Phi<2 \pi / 3$, which does not lead to frustration on triangular plaquettes. For such values of $\Phi$, the energies of all bonds can be simultaneously minimized on the whole triangular lattice, leading to a highly degenerate manifold of noncoplanar ground states. Our aim is to study the nature of ordering in the manifold of such states.

For the classification of all possible ground states, it is convenient to partition the triangular lattice into three equivalent triangular sublattices which are labeled $A, B$, and $C$ below. When the angles between neighboring spins are all equal to $\Phi$, neighboring spins on the same sublattice have either to be parallel to each other, or to make an angle $\Phi_{2}=2 \arccos \frac{\cos \Phi}{\cos (\Phi / 2)}$. It is convenient to say that in the latter case the spins are separated by a zero-energy domain wall, ${ }^{12}$ see Fig. 1(a). These domain walls live on the bonds of the honeycomb lattice composed by the sites of the remaining two sublattices (e.g., $B$ and $C$ if we consider domain walls for the spins on sublattice $A$ ), so that the bonds of the triangular lattice are partitioned into three distinct and nonoverlapping interpenetrating sets of bonds of three honeycomb lattices [consequently, we use three different colors to draw them, e.g., blue for walls passing through $A$ and $B$ sites, red for those passing through $B$ and $C$ sites and green for those passing through $C$ and $A$ sites (see Fig. 1)].

With this convention, any possible ground state can be characterized (or constructed) by specifying on some plaquette
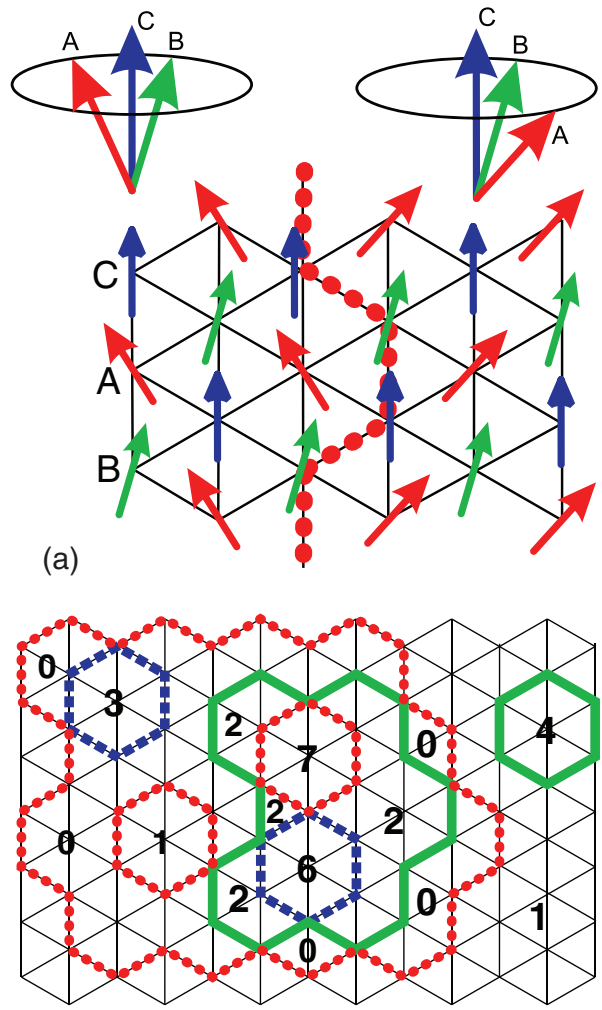

(b)

FIG. 1. (Color online) (a) Part of a triangular lattice with sublattices $A, B, C$, and a simple domain wall of type $A$ (on links $B C$ ) separating two domains having spins as represented by the two spin triads. The spins on sublattice $A$ differ in the two domains by the reflection with respect to the plane formed by the spins on the other two sublattices, which on both sides of the wall have the same orientation. (b) Visualization of a more complex domain wall configuration (without showing spins) giving rise to a set of domains of different regular states labeled (in an arbitrary way) by numbers from 0 to 7 .

three spins that form angles $\Phi$ with each other plus a configuration of domain walls. ${ }^{12}$ In this language, the simplest ground states in which all spins on the same sublattice have the same direction (the regular states with three-sublattice structure) correspond to the absence of domain walls. Any other ground state can be constructed from a regular state by introducing an appropriate set of domain walls on the aforementioned bonds of the honeycomb lattices.

For generic $\Phi$, the domain walls can end only at the boundary (or have to be closed) and cannot cross each other. Generic $\Phi$ here means

$$
\Phi \neq \pi / 2, \arccos (-1 / 3) .
$$

For these two specific values of $\Phi$, the local rules allow also for some other domain walls configurations which transform the model into an exactly solvable one. ${ }^{12}$ The analysis below always implies the fulfillment of condition (3).

The domain walls divide the system into domains of different regular states, see Fig. 1(b) for an example. Upon crossing a domain wall passing through sites $B$ and $C$, one reaches another domain in which the spins on sublattice $A$ are 
reflected with respect to the plane formed by the spins on two other sublattices, see Fig. 1(a). Similar rules hold for the other two types of domain walls. Summarizing, all ground states may be generated by systematically enumerating all possible domain wall configurations that exist on a lattice of a given size and by specifying which regular state is realized in one of the domains.

\section{B. Pseudospin representation}

In order to study the problem of ordering in noncoplanar states with Monte Carlo simulations, it is necessary to efficiently sample the space of allowed domain wall configurations. The simplest way to enumerate such configurations consists in introducing Ising-like (binary) variables $\sigma_{i}= \pm 1$ (pseudospins) defined on the sites of the same triangular lattice in such a way that each segment of a domain wall separates two pseudospins of opposite signs which are next-to-nearest neighbors of each other. It can be easily checked that the application of this rule does not lead to any contradiction for all allowed configurations of domain walls passing through a given site.

The existence of such a mapping allows one to replace the analysis of the loop model described in Sec. II A by that of the Ising-like model on a triangular lattice in which the only interaction of pseudospins consist in the interdiction to have intersections of domain walls, where domain walls are by definition the lines separating unequal pseudospins, which are next-tonearest neighbors of each other on the original triangular lattice (in other terms, nearest neighbors on the same sublattice). Remarkably enough, an Ising model with such an interaction has been introduced by Fendley, Moore, and $\mathrm{Xu}^{13}$ as a simple example of a two-dimensional model with a local geometric constraint without any reference to some physical system.

For us, the main practical advantage of using the pseudospin representation lies in its convenience for constructing efficient algorithms that sample the space of all domain-wall configurations allowed in the considered Heisenberg model, Eq. (1). In principle, these configurations can be then used to study correlations of the Heisenberg spins for any value of $\Phi$ from the interval $(0,2 \pi / 3)$. For other physical observables, an even more straightforward correspondence between the two models exists. Consider, for instance, the chirality $\chi_{k}$ defined as

$$
\chi_{k}=\operatorname{sign}\left\{\mathbf{S}_{j_{A}(k)} \cdot\left[\mathbf{S}_{j_{B}(k)} \times \mathbf{S}_{j_{C}(k)}\right]\right\}= \pm 1,
$$

where $j_{\alpha}(k)$ is the site of plaquette $k$ belonging to sublattice $\alpha$ (where $\alpha=A, B, C$ ). The chirality always changes sign upon crossing a domain wall, since one of the spins is flipped with respect to the plane formed by the other two spins. In terms of the pseudospin representation, the same quantity is given by

$$
\chi_{k}=\sigma_{j_{A}(k)} \sigma_{j_{B}(k)} \sigma_{j_{C}(k)},
$$

because now one of the pseudospins changes sign upon crossing a domain wall, while the other two are unchanged. This correspondence suggests that the chiral properties do not depend on the angle $\Phi$.

Even for the magnetic properties, for which there is no such a simple correspondence, it is possible to conclude on the existence of ordering by analyzing the properties of the pseudospin model. As discussed in Ref. 12, one expects that the presence of long-range correlations follows from having only a restricted number of uncontractable domain walls between two distant points on the lattice. Thus studying the statistics of loops (closed domain walls) in the pseudospin model allows one to reach qualitative conclusions regarding the behavior of spin-spin correlations in the original Heisenberg model, as done in Sec. V C.

In terms of the pseudospin representation, each regular state of the original Heisenberg model corresponds to one of the states in which on each sublattice all pseudospins are in a perfectly ordered state. It is clear that in total there exist only eight such states and that the infinite multitude of regular states, which can be achieved by successive spin flips on different sublattices, can be mapped onto these eight states. Thus each of the eight perfectly ordered states of the pseudospin representation may correspond to different regular states of the original model. Accordingly, the equivalence between the two models has some restrictions which are discussed in the next section.

\section{Boundary conditions and the Heisenberg sector}

The manifolds of all domain-wall configurations allowed by the local rules for the original Heisenberg model and for the pseudospin model are rigorously identical only when the system has a simple topology, i.e., lives on a simply connected lattice. However, when one imposes periodic boundary conditions, an important difference between the two models appears, as illustrated in Fig. 2.

This figure presents a configuration of pseudospins $\sigma_{j}= \pm 1$ with four domain walls crossing the whole system in the vertical direction. This configuration is perfectly compatible with having periodic boundary conditions both in the horizontal and vertical directions. However, in the case of Heisenberg spins, such a sequence of domain walls corresponds (when going from left to right) to successively flipping spins on sublattice $C$, then on sublattice $B$, then again on sublattice $C$, and finally again on sublattice $B$. Such a sequence of spin flips results in the rotation of the spins on the sublattices $C$ and $B$ by an angle $\Psi(\Phi)=2 \arccos \frac{\cos \Phi}{1+\cos \Phi}$ around the direction of the spins on sublattice $A .^{12}$ This means that in the generic case the regular states in the leftmost and

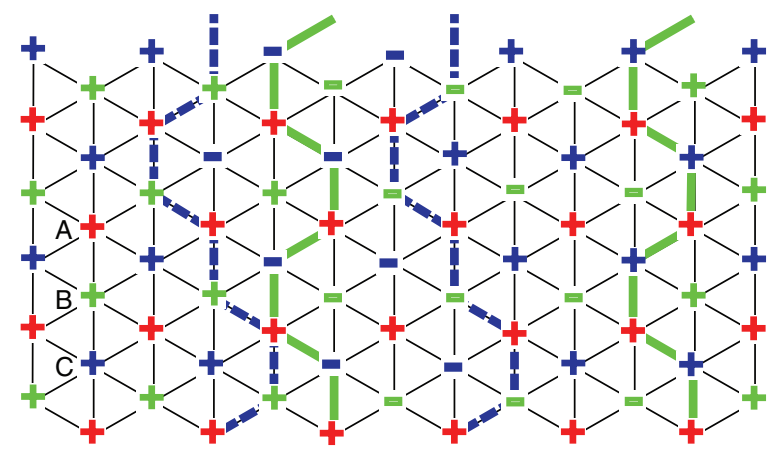

FIG. 2. (Color online) Example of a domain wall configuration (thick lines) in the pseudospin model with periodic boundary conditions which does not contradict the local rules but which is prohibited in the Heisenberg model. The values of the pseudospins are indicated by the plus and minus signs which are color coded according to the sublattice. 
rightmost domains of Fig. 2 are different. Therefore, in terms of the Heisenberg model, such a configuration of domain walls is not compatible with periodic boundary conditions in the horizontal direction.

Thus, in the case of periodic boundary conditions, the manifold of allowed domain wall configurations in the pseudospin model is larger than that of the original Heisenberg model. Below we refer to the set of domain wall configurations of the pseudospin model which are consistent with the periodic boundary conditions for Heisenberg spins as the Heisenberg sector of the pseudospin model. If the system has long-range order (as expected from the analysis of Ref. 12), the difference between the full partition function of the pseudospin model and that of its Heisenberg sector has to become negligible in the thermodynamic limit, since each state not belonging to the Heisenberg sector involves the presence of at least four domain walls crossing the whole system, while the presence of long-range order is incompatible with the existence of infinitely long domain walls.

Quite remarkably, the numerical analysis of the pseudospin model is automatically restricted to the Heisenberg sector if simulations are performed with the help of an algorithm based on local updates and are started from a perfectly ordered configuration. This is precisely the strategy which is applied in the next section. A more efficient algorithm involving sampling of all the states of the pseudospin model is introduced later, in Sec. IV.

\section{METROPOLIS MONTE CARLO SAMPLING OF GROUND-STATE MANIFOLD}

\section{A. Algorithm}

We will now describe the most basic Markov-chain Monte Carlo technique that allows to sample ground-state configurations in the pseudospin problem. The key observation is that in any ground-state configuration one can separate all pseudospins into flippable and unflippable. A pseudospin $\sigma_{j}$ at site $j$ is said to be flippable if flipping its sign leads to another ground state with nonintersecting domain walls. For this, there should be no domain wall passing through that site. On the other hand, pseudospins on sites passed through by domain walls are unflippable, because flipping any of them would create domain wall crossings which are prohibited. Naturally, each flippable (unflippable) pseudospin $\sigma_{j}$ corresponds to a flippable (unflippable) spin $\mathbf{S}_{j}$ in terms of the original Heisenberg model.

Accordingly, the simplest local Monte Carlo move one can imagine consists in changing the sign of a flippable pseudospin. In detail, our approach consists in defining a Monte Carlo sweep as a sequential scan through all the lattice sites with a flippability test of all the spins visited. If a spin is flippable, the Monte Carlo move of changing its sign is accepted with $50 \%$ probability. An alternative scheme would be to randomly choose spins and to always accept the move if a chosen spin is flippable. Note that both schemes satisfy detailed balance and are ergodic but have the obvious drawback of any local update-a slow displacement in the configuration space resulting in large autocorrelation times. On the other hand, this simple MC method has the convenient property that all pseudospin configurations generated by using local moves remain in the Heisenberg sector, if we start the Markov process from a regular state.

In the simulations, we construct the triangular lattice in the usual way by using a square lattice of linear size $L$ (containing $N=L^{2}$ sites) with diagonals along one direction. Periodic boundary conditions are applied with respect to the base square lattice. In such a setup, in order to keep the three-sublattice structure, $L$ has to be a multiple of 3 . We have verified the correctness of our algorithm by comparing its results with those of an exact enumeration approach in which we systematically generated all possible ground states in small systems up to the one containg $N=6^{2}$ spins (which in the case of periodic boundary conditions has exactly 2176672 degenerate ground states).

\section{B. Magnetization and chirality}

In order to study whether a typical state belonging to the ground-state manifold is ordered, we turn to an estimation of physical observables (expressed in terms of the pseudospins) using the MC sampling procedure. The basic magnetic quantities studied below are the sublattice magnetizations

$$
m_{\alpha}=\frac{3}{N} \sum_{i \in \mathcal{T}_{\alpha}} \sigma_{i},
$$

where $\alpha \in\{A, B, C\}$ denotes a particular triangular sublattice and $\mathcal{T}_{\alpha}$ is the set of all sites belonging to this sublattice. In any state with perfectly ordered sublattices, these magnetizations are equal to \pm 1 resulting in an eightfold degeneracy in accordance with the number of the possible combinations of signs. In order to verify if the same order parameter survives in a typical ground state, we also analyze the size dependence of the mean of the absolute values of the three sublattice magnetizations,

$$
m_{s}=\frac{1}{3} \sum_{\alpha}\left|m_{\alpha}\right|,
$$

as well as the analogous mean of the squared sublattice magnetizations. In a state in which at least one sublattice has a long-range order, both $\left\langle m_{s}\right\rangle$ and $\left\langle m_{\alpha}^{2}\right\rangle$ will be nonvanishing and $\left\langle m_{\alpha}^{2}\right\rangle$ can be expected to converge to the same limit as the spin-spin correlation function $\left\langle\sigma_{i} \sigma_{j}\right\rangle$, where $i$ and $j$ are sites on the same sublattice maximally distant from each other. Here and below, angular brackets denote the standard ensemble average, which is calculated by Monte Carlo sampling.

We also consider the chirality $\chi$ defined by

$$
\chi=\frac{1}{N} \sum_{k \in \Delta} \chi_{k},
$$

with $\chi_{k}$ the chirality on plaquette $k$ as defined in Eqs. (4) and (5). The sum in Eq. (8) runs over all $N=L^{2}$ up triangular plaquettes of the lattice (the sum over down plaquettes has the same value).

\section{Simulation results for magnetization and chirality}

We have calculated the above quantities for various linear sizes between $L=12$ and $L=192$ (as explained in Sec. III A, $L$ has to be a multiple of 3 ). In each case 
we took $10^{5}$ independent measurements. Independence of the measurements was achieved by calculating observables only for every $L^{2}$ generated configurations, reflecting the increase of autocorrelation time $\tau \sim L^{2}$ found for the sampling scheme (from time series of the magnetization and the chirality). The overall time complexity of the local algorithm is thus of the order of $O\left(L^{4}\right)$.

Since in any perfectly ordered state (without domain walls) the normalized chirality defined by Eq. (8) is equal to plus/minus one, a study of $\langle|\chi|\rangle$ should be one of the most direct and simple ways to check if a typical ground state is ordered as we expect. However, our MC results for the chirality, shown in Fig. 3(a), do not confirm this expectation since they do not show any tendency for the convergence to a finite value. On the contrary, these data rather accurately follow the straight line corresponding to a $\langle|\chi|\rangle \propto 1 / L$ decay of the chirality with the system size.

Note that this dependence should not be taken as a sign of algebraic correlations of $\chi_{k}$. Indeed, the same dependence would be obtained if the chiralities of different plaquettes were simply uncorrelated from each other. Thus the observed size dependence of $\langle|\chi|\rangle$ suggests that the ground states are disordered in terms of chirality, or, at most, have a very small

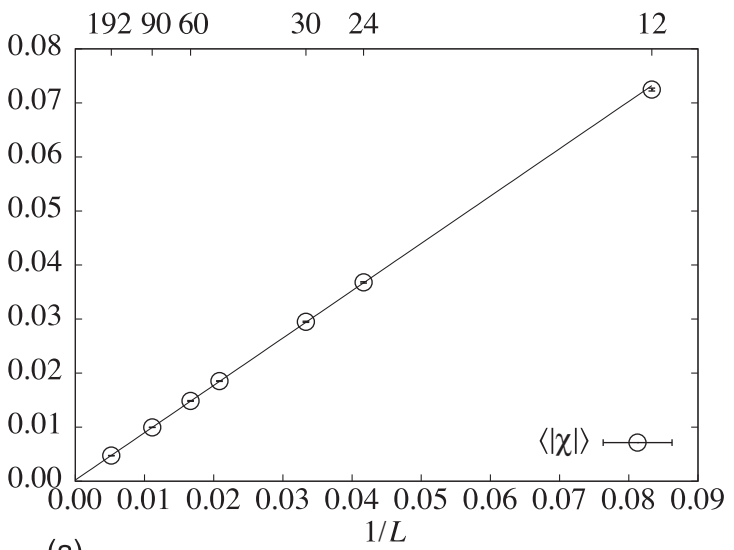

(a)

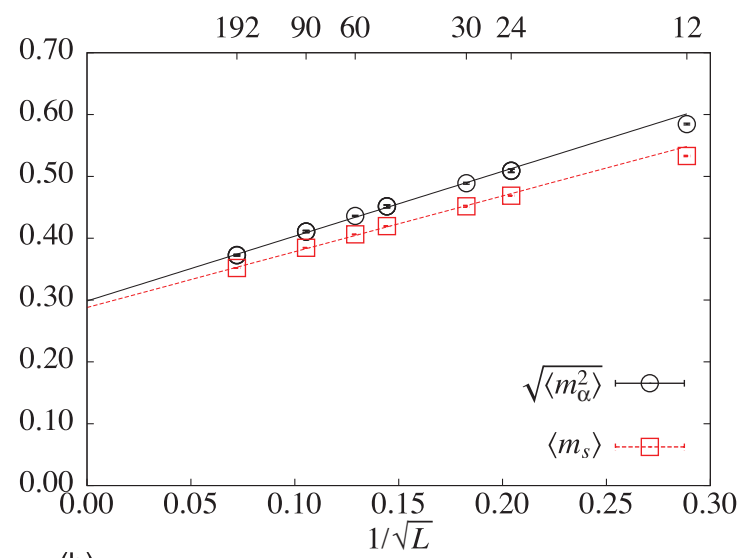

(b)

FIG. 3. (Color online) (a) The chirality $\langle|\chi|\rangle$ tends to zero like $1 / L$. (b) Size dependence of the average sublattice magnetization $\left\langle m_{s}\right\rangle$ and of $\sqrt{\left\langle m_{\alpha}^{2}\right\rangle}$. In the thermodynamic limit, both quantities extrapolate to a same finite value with corrections that vanish approximately like $1 / \sqrt{L}$. Here, as well as in the subsequent plots, error bars are shown but are smaller than the symbol size. chirality whose resolution is beyond the limits of the method used in this section.

In evident contrast to this finding, the probe for ordering based on the sublattice magnetization is clearly in favor of an ordered situation. A finite-size analysis of both $\left\langle m_{s}\right\rangle$ and $\sqrt{\left\langle m_{\alpha}^{2}\right\rangle}$, shown in Fig. 3(b), indicates the presence of a longrange ordering with both quantities converging to the same finite value of the spontaneous magnetization $\left\langle m_{s}\right\rangle \approx 0.29$ in support of the conclusions of Ref. 12. This extrapolation is based on assuming that the finite-size corrections decay like $L^{-1 / 2}$, as suggested by the data presented in Fig. 3(b). The value of the magnetization is naturally suppressed compared to the totally ordered (regular) states. Still, under the assumption that in the ordered state all three sublattices remain equivalent, the simplest estimate assuming $\langle|\chi|\rangle \sim\left\langle m_{s}\right\rangle^{3}$ would suggest for the chirality a detectable value $\langle|\chi|\rangle \sim 0.024$, which is clearly not observed.

Motivated by this lack of consistency, we decided to have a more attentive look at the distribution and the size dependence of individual sublattice magnetizations to check if in a typical ground state, the three sublattices are equivalent, that is have (up to a sign) the same magnetization in the thermodynamic limit. Any other situation would mean the presence of an additional discrete degeneracy related to the breaking of the symmetry with respect to the permutation of sublattices. To this end, we record for each ground-state configuration the triad of sublattice magnetizations $\left\{m_{A}, m_{B}, m_{C}\right\}$ and sort them into an ordered triad $\left\{m_{1}, m_{2}, m_{3}\right\}$ with $\left|m_{1}\right| \leqslant\left|m_{2}\right| \leqslant\left|m_{3}\right|$. Figure 4(a) shows MC results for the sorted magnetizations, which make apparent that the sublattice order is to a large degree heterogeneous: typically, two of the three sublattices show ordering correlations, while the third has a much smaller magnetization together with a finite-size scaling trend, which seems incompatible with an ordered state in which all three sublattices order (up to the sign) equally. This is also evident in Fig. 4(b) showing typical distribution functions of the sorted magnetizations $\left|m_{1}\right|,\left|m_{2}\right|$, and $\left|m_{3}\right|$ for $L=192$, indicating that $m_{1}$ has a very prominent support (large weight) in the region of disordered configurations.

These data are clearly insufficient to understand whether (i) a typical ground state is partially ordered with one sublattice becoming completely disordered in the thermodynamic limit (leading to a state trivially consistent with a vanishing chirality), or (ii) when $L$ increases, the minimal magnetization $m_{1}$ will eventually reverse its trend and converge towards the same limit as $m_{2}$ and $m_{3}$, or (iii) some other (intermediate) scenario is realized. In any case, the question whether a finite chirality is ultimately stabilized seems primarily linked to the fate of the smallest sublattice magnetization $m_{1}$ in the thermodynamic limit and the ambiguity in the current data calls for simulations on much larger systems in order to resolve this question. The remainder of this paper is focused on the construction of an efficient algorithm allowing to undertake such simulations as well as on its implementation for a numerical resolution of the puzzle just described.

\section{CLUSTER MONTE CARLO ALGORITHM}

In the local MC procedure used in Sec. III, the updates were restricted to individual pseudospins which can be flipped 


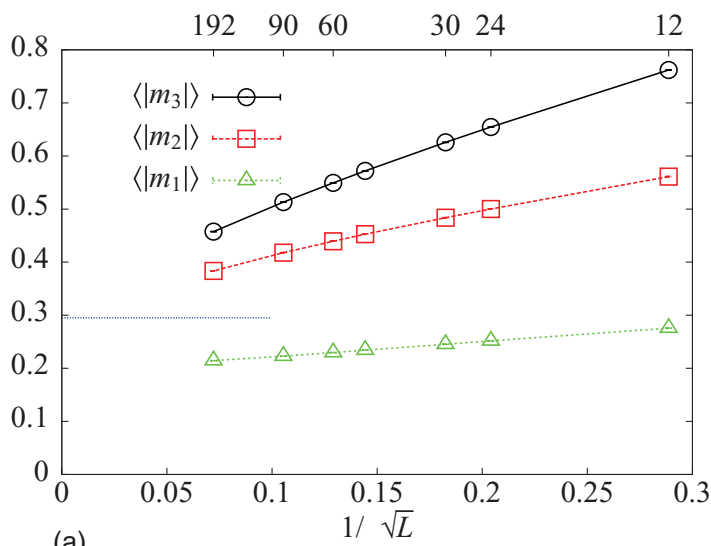

(a)

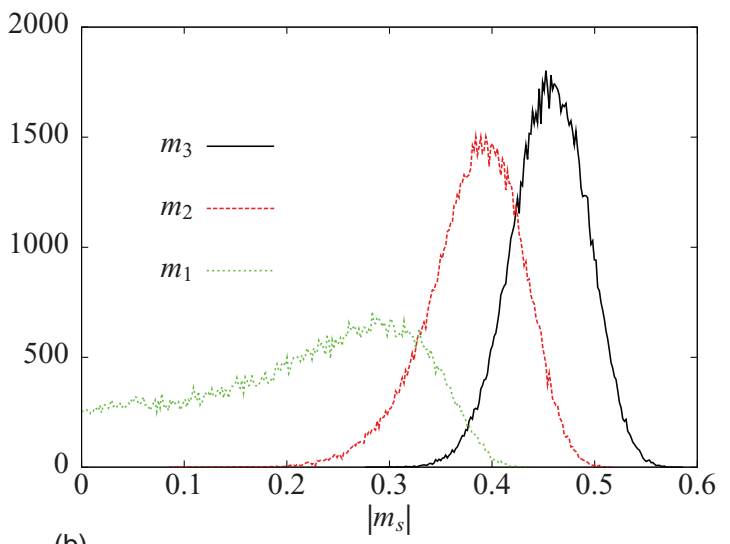

(b)

FIG. 4. (Color online) (a) Size dependence of the three sorted sublattice magnetizations $\left\langle\left|m_{1}\right|\right\rangle,\left\langle\left|m_{2}\right|\right\rangle$, and $\left\langle\left|m_{3}\right|\right\rangle$. While the behavior of $\left\langle\left|m_{3}\right|\right\rangle,\left\langle\left|m_{2}\right|\right\rangle$ is consistent with an extrapolation towards the average sublattice magnetization (shown with a dashed horizontal line), the behavior of $\left\langle\left|m_{1}\right|\right\rangle$ is puzzling. Lines are included as a guide to the eye. (b) Distribution functions of the sorted sublattice magnetizations encountered in ground-state configurations for $L=192$, indicating the frequent presence of partially disordered configurations.

without creating domain walls crossings. Such flippable spins are easily recognizable because they sit on the sites which are not passed through by any domain wall. Locally flipping an unflippable spin (sitting on a site belonging to some domain wall) would result in a violation of the constraint that domain walls cannot cross. It is, however, possible to flip an unflippable pseudospin by flipping a whole cluster of unflippable pseudospins from the same sublattice, and it will be natural to call such a cluster a flippable cluster. A flippable pseudospin can be considered as the simplest example of a flippable cluster. The first nontrivial example of a flippable cluster (e.g., on sublattice $\mathcal{T}_{A}$ ) consists of all pseudospins on $\mathcal{T}_{A}$ that are part of, say, an $A B$ loop not touching another domain wall, see Fig. 5(a). To avoid the intersections of domain walls, all pseudospins on $\mathcal{T}_{A}$ visited by the same wall have to be equal to each other. Naturally, after being flipped they remain equal to each other, which further ensures the absence of wall crossings.

Flippable clusters have a more complicated structure when domain walls touch each other. Consider for instance a particular $A B$ loop touching an $A C$ loop (or a few of them). To

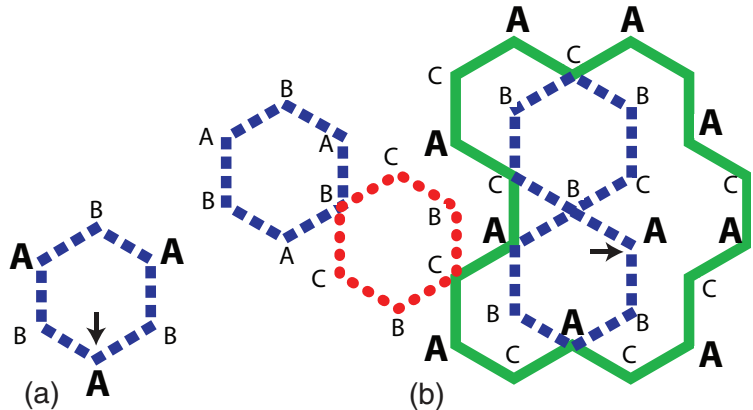

FIG. 5. (Color online) Visualization of flippable clusters of pseudospins. (a) A simple cluster of A spins (shown in bold) within an $A B$ loop that does not touch any other loops. (b) A more complicated cluster of A spins (shown in bold) in a situation where multiple domain walls touch each other. Note that not all $A$ sites of the domain wall network belong to the flippable cluster. In both panels, the site from which the construction of the flippable cluster is started is indicated by an arrow.

ensure the absence of wall crossings, one has to flip not only all $\mathcal{T}_{A}$ pseudospins belonging to a chosen $A B$ loop, but also all $\mathcal{T}_{A}$ pseudospins belonging to the $A C$ loops touching it, and also all $\mathcal{T}_{A}$ pseudospins belonging to all $A B$ loops touching these $A C$ loops and so on. In other words, one has to flip $\mathcal{T}_{A}$ pseudospins on the whole connected cluster formed by $A B$ and $A C$ loops touching each other, as indicated in Fig. 5(b). Note that in terms of the original Heisenberg model all pseudospins belonging to the same flippable cluster correspond to equal spins $\mathbf{S}_{i}$.

Using this approach, a valid global update can be constructed applying a single cluster technique in the spirit of the Wolff algorithm, ${ }^{14,15}$ but with completely deterministic cluster construction: (1) pick a random site $i$ (and determine its sublattice index $\alpha$, say $A$ ), (2) check if spin $\sigma_{i}$ is flippable and flip it if it is, and (3) if $\sigma_{i}$ is unflippable, construct the connected cluster of domain walls formed by $A C$ and $A B$ loops and flip all the spins on sublattice $A$ belonging to that cluster.

The construction of connected clusters can be done purely recursively in a depth-first approach which is usually the most convenient choice to code. However, this technique requires large stack memory resources for big clusters which can be a severe limitation. In view of the large system sizes that are required in this study, we have opted for a hybrid cluster identification approach in the spirit of Ref. 16 in which we follow domain walls iteratively until a touching point with another domain wall is found in which case we continue cluster construction recursively. Such an approach is expected to be less demanding with respect to stack memory. In our implementation, a typical Monte Carlo sweep then consists of one pass through the lattice where we check for flippable spins and apply the local update. This sweep is followed by $N_{\text {sc }}$ single-cluster updates where $N_{\text {sc }}$ is an integer number such that on average say $N / 3$ sites are touched during the cluster construction (in an accumulated way). This number $N_{\mathrm{sc}}$ is determined self-consistently during the equilibration phase of the algorithm. With this setup, the nonlocal update turns out to be highly efficient with no sign of critical slowing down at all: 
the dynamic exponent $z$ [measured from the size dependence of the autocorrelation time $\tau(L) \sim L^{z}$ for the total magnetization $\left.m=\sum_{\alpha} \sigma_{\alpha}\right]$ cannot be distinguished from zero. Essentially, this means that the algorithm generates independent configurations with time complexity $O\left(L^{2}\right)$ compared to $O\left(L^{4}\right)$ for the local update.

\section{SIMULATION RESULTS}

Using the cluster algorithm, we have investigated much larger systems than before going up to $L=11340$, which is roughly 50 times larger than could be done with the local update. In detail, we studied systems of linear sizes $L=384,576,1152,2304,3456,4608,5760$, and 11340 next to those already considered in the previous section (the total number of spins $N$ being $L^{2}$, there were 128595600 spins in the largest cluster). Typically, we collected of the order of $10^{6}$ independent measurements (for the largest system sizes $10^{5}$ ) in each case.

\section{A. Winding loops and the Heisenberg sector}

As already mentioned in Sec. II C, a special property of the cluster update (in contrast to the local MC procedure) is that in a system with periodic boundary conditions it can generate pseudospin configurations which do not correspond to any ground state of the original Heisenberg model with the same boundary conditions (in the case of free boundary conditions, this discrepancy does not exist). All such states are characterized by the presence of winding domain walls, that is, of incontractible loops, which wind around the torus. However, not all ground states containing some sequence of winding domain walls correspond to the violation of the periodic boundary condition in the Heisenberg model. Some sequences of winding domain walls (which below are called reducible) can be obtained as well in the framework of local update and the states containing them represent true ground states of the Heisenberg model.

In order to check if a given pseudospin configuration belongs to the Heisenberg sector or not (in the latter case it contains an irreducible sequence of winding domain walls), the following simple procedure can be used. Let us denote a sequence of domain walls crossed by a closed path going around one of the two directions on the torus by a word $w$, for example, $w=$ CABBAC, in which each letter stands for the type of domain wall crossed by the path. Namely, letter A stands for crossing a loop consisting of segments along $B C$ links, $\mathrm{B}$ for segments along $C A$ links, and $\mathrm{C}$ for $A B$ type wall. A simple check whether the configuration is compatible with the Heisenberg sector consists in testing whether the word w obtained in such a way can be reduced to an empty word by repeatedly applying the rule that two successive letters in the word annihilate themselves if they are identical. The possibility to get rid of two identical letters when they are adjacent originates from the possibility to annihilate a pair of neighboring domain walls of the same type by making local single-spin flips on the corresponding sublattice between them. For instance, the word $\mathrm{w}=\mathrm{CABBAC}$ can be reduced to the empty word (since $\mathrm{CABBAC} \rightarrow \mathrm{CAAC} \rightarrow \mathrm{CC}$ ) whereas the word $\mathrm{CBCB}$, obtained from Fig. 2 when going along a closed path in the horizontal direction, cannot and is thus said to be irreducible. A configuration belongs to the Heisenberg sector if for both directions on the torus the procedure described above produces empty words.

Below, the fraction of configurations outside the Heisenberg sector is denoted by $p_{\mathrm{nH}}$ and the fraction of configurations with reducible sequences of winding domain walls by $p_{\mathrm{w}}$. An analysis of both quantities is of interest as in general all sequences of winding domain walls generate competing strips of domains spanning the whole system. Hence even the Heisenberg sector can suffer from considerable finite-size effects due to the presence of reducible sequences of winding domain walls. In particular, since domain walls cannot cross each other, the winding domain walls have to choose one of the two preferred directions imposed by periodic boundary conditions and therefore their presence makes a configuration essentially anisotropic.

First, for the configurations outside the Heisenberg sector, the simulations undoubtedly demonstrate that $p_{\mathrm{nH}}$ very quickly drops to zero, see Fig. 6. Therefore these configurations

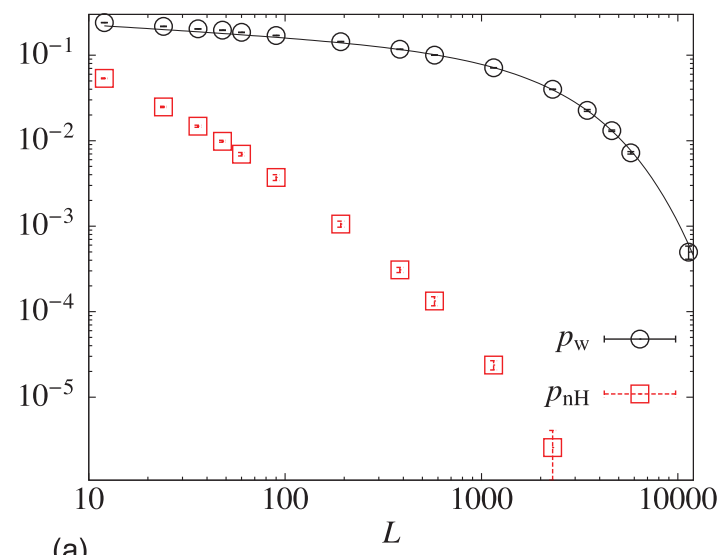

(a)

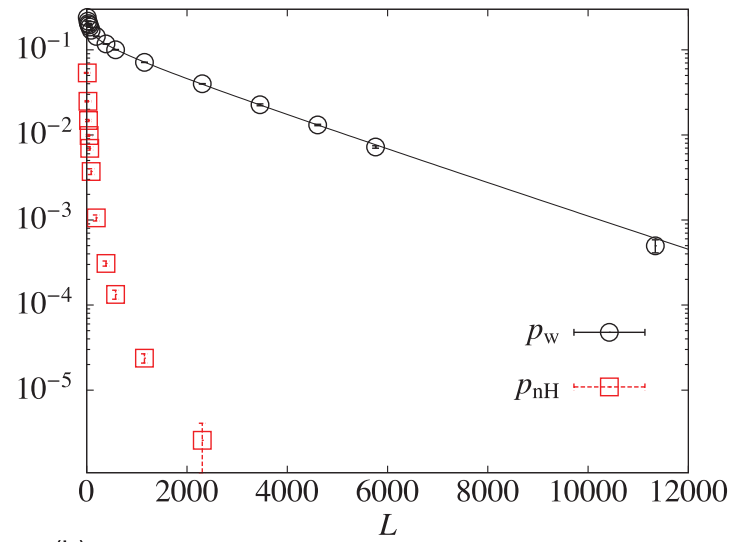

(b)

FIG. 6. (Color online) The proportion of domain-wall configurations that are inconsistent with the Heisenberg sector $\left(p_{\mathrm{nH}}\right)$ quickly drops to zero as a function of lattice size as shown in a double logarithmic plot (a) and in a semilogarithmic plot (b). For $L>2000$, no such configurations were observed within the simulation time. On the other hand, a much larger fraction $p_{\mathrm{w}}$ of configurations belonging to the Heisenberg sector but incorporating winding loops is observed to decay much more slowly. For sizes $L \gtrsim 1000$ the behavior of $p_{\mathrm{w}}$ is consistent with an exponential decay as evidenced in the semilogarithmic plot. 


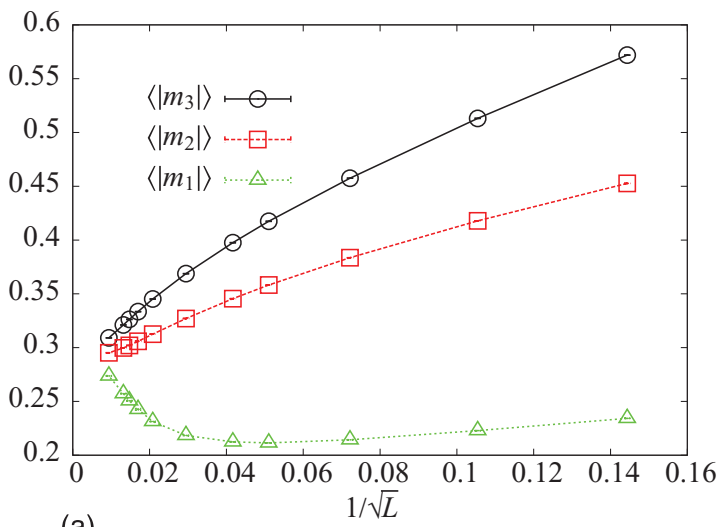

(a)

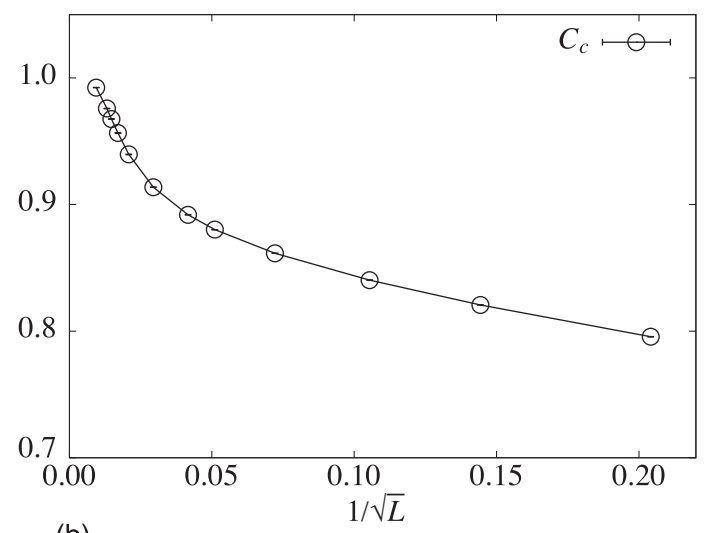

(b)

FIG. 7. (Color online) (a) Plot of the sorted sublattice magnetizations for $L$ up to 11340 demonstrating that the three sublattices become equivalent in the thermodynamic limit. (b) $C_{c}$ defined by Eq. (9) provides a quantitative measure allowing one to characterize the nature of ordering. Its convergence to 1 (the upper boundary for $C_{c}$ ) indicates the tendency of the sublattice magnetizations to become equal to each other. The scale $1 / \sqrt{L}$ is somewhat arbitrary and has been motivated by the size dependence of the magnetization at small scales.

have no effect whatsoever on physical quantities in the thermodynamic limit and the results obtained in the framework of cluster update are applicable also for the description of the original Heisenberg model. Even in small systems with $L=12$, where we find a considerable fraction (about $\approx 0.1$ ) of such non-Heisenberg configurations, the systematic error made on estimating physical quantities without filtering out the non-Heisenberg configurations is only of the order of $0.4 \%$ for the chirality and $1.8 \%$ for the sublattice magnetization. Naturally, restricting the analysis to the Heisenberg sector, we recover the same results as with the local update.

A finite-size analysis of $p_{\mathrm{w}}$, also shown in Fig. 6, highlights the fact that the fraction of configurations with reducible sequences of winding domain walls remains considerable up to very large system sizes, with a rather slow decrease described initially by an algebraic-like behavior with a small effective exponent $b \approx 0.2$. For lengths greater than $\sim 1000$, the decrease, however, is consistent with an exponential decay $\sim \exp (-L / \lambda)$. We determine this length scale to be given by $\lambda \approx 2000$. The exponential decay of $p_{\mathrm{w}}$ suggests that all domain walls have a finite free energy per unit length, which is compatible with having a true long-range order on all three sublattices.

\section{B. Solving the ordering puzzle}

We are now in a better position to readdress the puzzling situation described in Sec. IIIC, that is, to study whether the noncoplanar ground states have true long-range order (in magnetization and in chirality), are only partially ordered, or if some entirely different scenario is realized. To learn more on the nature of sublattice ordering, we start by extending the analysis of the size dependence of the ordered triad of magnetizations $\left\{m_{1}, m_{2}, m_{3}\right\}$ (presented for $L \leqslant 192$ in Fig. 4) to larger systems. The new data, shown in Fig. 7, immediately confirm the need for simulations on large $L$ : the behavior of the smallest magnetization $m_{1}$, one of the main puzzles of the local update results, turns out to be nonmonotonous. This quantity passes through a minimum (situated roughly at $L \sim 500$ ) and after that it ultimately tends towards a common limit with $m_{2}$ and $m_{3}$. This finding definitely speaks in favor of a situation in which all three sublattices are ordered to an equal degree.

To investigate this question more quantitatively, we study the ratio,

$$
C_{c}=\frac{3\left(m_{1}^{2} m_{2}^{2}+m_{1}^{2} m_{3}^{2}+m_{3}^{2} m_{2}^{2}\right)}{\left(m_{1}^{2}+m_{2}^{2}+m_{3}^{2}\right)^{2}},
$$

of the two quartic invariants normalized in such a way that $C_{c}$ always belongs to the interval $[0,1]$. In the thermodynamic limit, $L \rightarrow \infty, C_{c}$ has to tend to 1 for an ordered state in which all sublattices are, up to the sign, equally ordered, to $3 / 4$ for a partially ordered state in which two sublattices are ordered equally and the third one is disordered, and to 0 when only one sublattice is ordered. The size dependence of $C_{c}$ demonstrates equal strength sublattice ordering but again clearly points to the difficulty of the problem: while the data for $L<500$ could be interpreted as tending to a value around 0.9 , which would imply that all three sublattices are ordered, but have different magnetizations, a relatively abrupt change of regime takes place at a particular length scale with convergence to the state in which all sublattices are equally ordered at larger $L$.

Having thus constrained the possible ordering scenario, the next nontrivial question is whether the ground-states are characterized in the thermodynamic limit by a nonvanishing chirality $\chi$. Naturally, we used the large-scale data also to study the behavior of the chirality, as well as of the averaged sublattice magnetization to reach a precise quantitative characterization for the degree of ordering. The numerical data for $\langle|\chi|\rangle$, shown in a double logarithmic plot in Fig. 8, provide the second key result of this section. They unambiguously demonstrate the chiral nature of the noncoplanar ground states, whereas the finite-size behavior again shows the nontrivial features characteristic for this study: over three decades in $L$, the decay of chirality very accurately follows an algebraic law $\langle|\chi|\rangle \sim L^{-1}$ [consistent with the observation in Fig. 3(b)] before overcoming a length scale upon which a very small but finite expectation value is reached. Essentially, this situation is reminiscent of very weak ordering close to a critical point.

Before analyzing this situation in more detail, we note that a similar observation holds for the sublattice magnetization (concentrating here on the quantity $\left\langle m_{s}\right\rangle$ ) which is saturating 


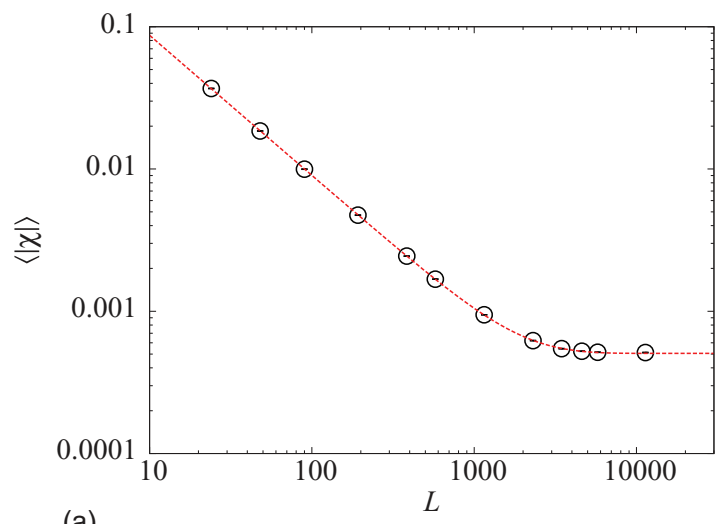

(a)

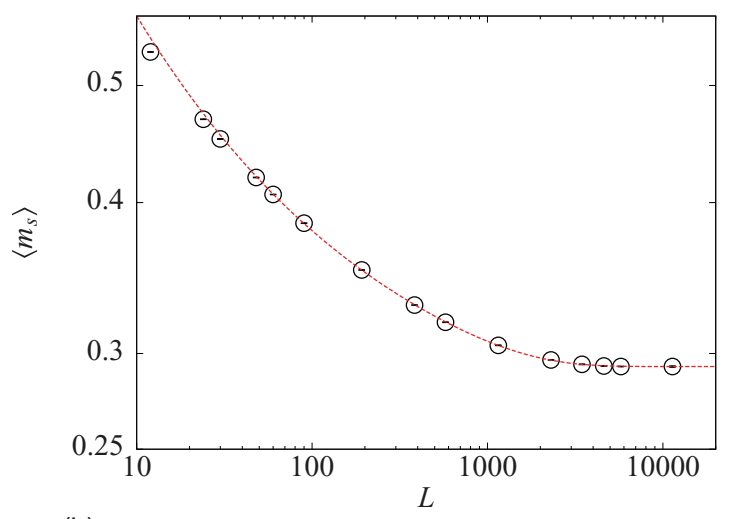

(b)

FIG. 8. (Color online) Plot of (a) the chirality and (b) the sublattice magnetization in a double logarithmic scale covering the complete range of lattice sizes simulated. Both quantities show a convergence to their infinite-size values for the largest systems studied. Dashed lines are results of fits to Eq. (10).

above a certain length scale and that the system sizes studied are now sufficiently large to represent the thermodynamic limit: the estimates for the sublattice magnetization for $L=5760$ and 11340 agree within the error bar, and take the value $\left\langle m_{s}\right\rangle \approx 0.2926$. This reinforces the observation that we have reached a length scale in our simulations where the effective finite-size corrections are no longer algebraic, but rather have a conventional exponential form $\sim \exp (-L / \lambda)$, where $\lambda$ is a length scale proportional to the correlation length.

In order to extrapolate the data as well as to characterize and capture the corrections over a large window of system sizes, a reasonable ansatz for the finite-size dependence of some observable $A$ is

$$
\langle A\rangle=\langle A\rangle_{\infty}+a L^{-b} \exp (-L / \lambda),
$$

where $b$ is an effective exponent and $a$ some constant coefficient. Note that this form has been chosen just as an effective means of extrapolating and describing our results, but we are not aware of a particular theory predicting the finite-size dependencies of quantities of interest for the present model. For the conventional Ising model on a square lattice, we have verified numerically that the extrapolation of the magnetization using Eq. (10) close to criticality gives excellent agreement with exact results.

Table I shows some results obtained from fits for various fitting windows $L \in\left[L_{\min }, L_{\max }\right]$ with fixed $L_{\max }=11340$.
TABLE I. Results of extrapolations of the magnetization and chirality based on Eq. (10) for fitting windows with different $L_{\min }$. In the both parts of the table the two last lines show the parameters of a purely exponential fit with $b=0$.

\begin{tabular}{lccccc}
\hline \hline$L_{\min }$ & $\left\langle m_{s}\right\rangle_{\infty}$ & $\lambda$ & $a$ & $b$ & $\chi^{2} /$ d.o.f \\
\hline 100 & $0.29252(9)$ & $1413(40)$ & $0.96(3)$ & $0.50(1)$ & 1.2 \\
200 & $0.29252(10)$ & $1412(50)$ & $0.95(5)$ & $0.50(2)$ & 0.7 \\
500 & $0.29253(10)$ & $1362(116)$ & $0.81(20)$ & $0.47(6)$ & 0.5 \\
1000 & $0.29259(8)$ & $986(21)$ & $0.039(1)$ & $\ldots$ & 0.8 \\
2000 & $0.29252(10)$ & $1088(50)$ & $0.031(3)$ & $\ldots$ & 0.4 \\
$L_{\min }$ & $\langle|\chi|\rangle_{\infty}$ & $\lambda$ & $a$ & $b$ & $\chi^{2} /$ d.o.f \\
200 & $0.000511(3)$ & $1546(50)$ & $0.66(5)$ & $0.94(2)$ & 5.3 \\
500 & $0.000512(1)$ & $1471(60)$ & 0.5 & $0.87(2)$ & 2.5 \\
1000 & $0.000515(3)$ & $1000(60)$ & $0.0017(1)$ & $\ldots$ & 10.0 \\
2000 & $0.000513(1)$ & $959(20)$ & $0.00140(5)$ & $\ldots$ & 0.57 \\
\hline \hline
\end{tabular}

Error bars were obtained using a bootstrap approach where fits were repeated several times varying the data within their statistical uncertainty. In the case of the sublattice magnetization, very good fits - in terms of the standard chi-square test ${ }^{17}$ for the variance of residuals per degree of freedom $\left(\chi^{2} /\right.$ d.o.f $\left.\sim 1\right)$-are obtained describing the data over a wide range of system sizes. For $\left\langle m_{s}\right\rangle$, the parameter $\lambda$ turns out to be $\lambda \approx 1500$, quantifying thus the order of magnitude of system sizes to be overcome in the simulations, and the effective exponent $b \approx 0.55$ agrees well with the approximate finite-size behavior $L^{1 / 2}$ observed in the simulations using purely local Monte Carlo moves. In addition to fits to Eq. (10), we have performed fits using purely exponential corrections $(b=0)$, which work very well for the largest system sizes $L>1000$ but do not capture finite-size corrections for smaller sizes, see Table I. Results from purely exponential fits give consistent values for the sublattice magnetization and a slightly smaller $\lambda \approx 1000$.

Similarly, the finite-size dependence of the chirality is well described by $\lambda \approx 1500$ and $b \approx 0.9$ [Eq. (10) for $L>200$ ] and $\lambda \approx 1000$ (purely exponential fit for $L>2000$ ) but the quality of the fits is generally less good than for the magnetization indicating that additional finite-size effects are likely to be present. However, the finite-size extrapolated value of the chirality is rather stable and consistent between all the fits.

In summary, the large-scale simulations in the present section reveal not only the nature of magnetic order (with equal magnetization of all three sublattices) in a typical ground state but also establish the chiral nature of such a state, essentially resolving the puzzling situation described in Sec. III. Our final estimates for the infinite-size local order parameters characterizing the order in the pseudospin model are

$$
\begin{gathered}
\left\langle m_{s}\right\rangle_{\infty}=0.2925(2), \\
\langle|\chi|\rangle_{\infty}=0.000513(5),
\end{gathered}
$$

where the error bar is a conservative estimate (twice as large as the largest error bars from the fits). Interestingly, the chirality turns out to be reduced by two orders of magnitude compared to the naive estimate $\left\langle m_{s}\right\rangle_{\infty}^{3} \approx 0.025$ indicating the presence of large fluctuations. 


\section{Irreducible sequences of domain walls, distribution on the Cayley tree, and correlation functions}

The cluster update has enabled us to conclude on the nature of ordering in the pseudospin model by obtaining a precise characterization of the magnetic order as well as by proving the chiral nature of a typical ground state. Since the chirality can be also defined in terms of the Heisenberg spins, the latter conclusion directly applies to the original Heisenberg model, but the relation between magnetic properties of the two models is less straightforward. In this Section, we suggest an approach to characterize quantitatively fluctuations of the Heisenberg model in its full order-parameter space (without reduction to the pseudospin representation) and use it to draw conclusions directly on the magnetic ordering of Heisenberg spins.

Following the presentation of Ref. 12, we remind the reader that the relationship of any two regular spin domains can be characterized by the shortest sequence of domain walls one has to cross in order to get from one domain into the other (the irreducible domain wall sequence), or in other terms, by sequentially applying the spin flips associated with the types of domain walls separating them. For two given plaquettes on the triangular lattice, $k_{1}$ and $k_{2}$, this sequence is easily obtained by connecting these plaquettes by an arbitrary path on the dual lattice and constructing a word $w$ from letters A, B, C in accordance with the types and order of domain walls which are crossed along this path. Following the discussion of Sec. V A, this word has then to be simplified iteratively applying the rule that any neighboring identical letters annihilate each other until no further simplification is possible. The irreducible word $\mathbf{w}_{k_{1} ; k_{2}}$ obtained in such a way is unique (i.e., it does not depend on the particular path connecting $k_{1}$ and $k_{2}$ ) as soon as the ground-state configuration belongs to the Heisenberg sector. The subsequent analysis is thus restricted to this sector, although in the thermodynamic limit this is of no importance, as already discussed in Sec. V A.

Because in an irreducible domain wall sequence adjacent walls have to be of different types the manifold of all irreducible domain wall sequences has the structure of a tree, namely that of an infinite Cayley tree with coordination number 3. This means that when considering a particular ground state, one can characterize the position of any domain in the order parameter space by a node on this tree. Accordingly, the length of the irreducible word, $d=\left|\mathrm{w}_{k_{1} ; k_{2}}\right|$, naturally defines the distance between the two domains in the order parameter space (that is, on the Cayley tree). Arguments that this distance remains finite regardless of the spatial distance of the domains was one of the key steps towards making the case for the existence of magnetic ordering in the noncoplanar ground states in Ref. 12 .

In order to address this point numerically, we consider plaquettes $k$ and $k^{\prime}(k)$ that are maximally separated on the triangular lattice with periodic boundary conditions [that is are transformed into each other by a shift by a vector $\mathbf{T}=(L / 2, L / 2)]$ and calculate the average distance $d_{\mathrm{av}}$ on the Cayley tree between the domains to which they belong by using

$$
d_{\mathrm{av}}=\frac{1}{N} \sum_{k}\left|\mathrm{w}_{k ; k^{\prime}(k)}\right|,
$$

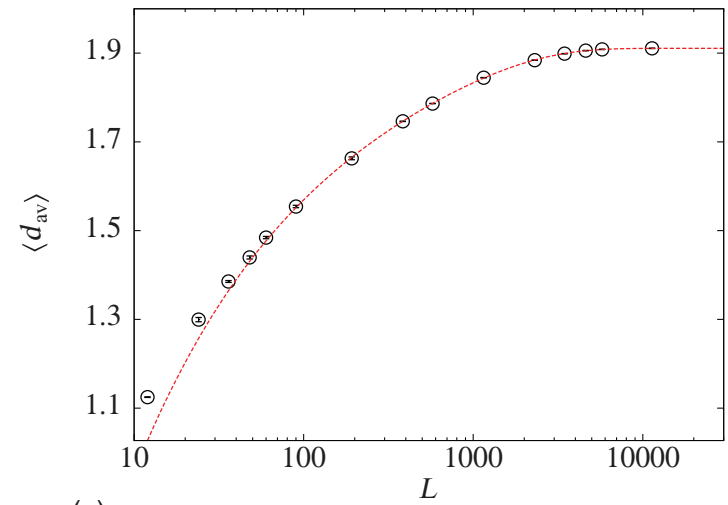

(a)

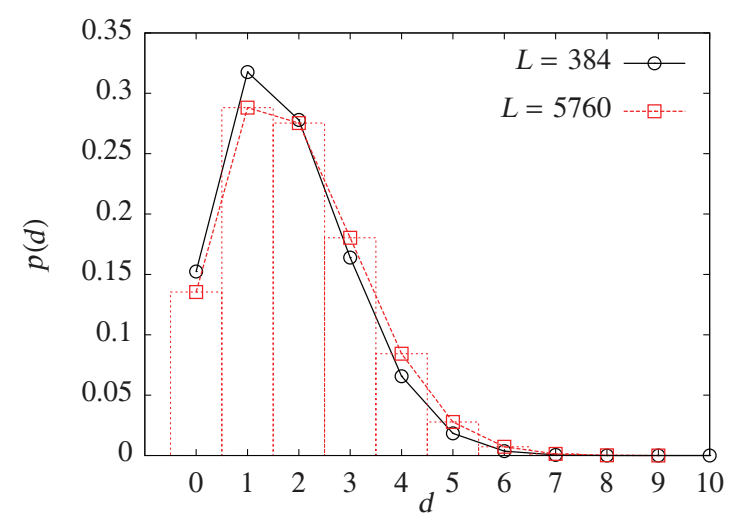

(b)

FIG. 9. (Color online) (a) Size dependence of the average number of irreducible domain walls between two plaquettes $k$ and $k^{\prime}(k)$ situated at the maximal possible distance from each other. For very large system sizes, a convergence is evident. The dashed line is a fit to the ansatz given by Eq. (10). (b) Distribution function $p(d)$ of the number of irreducible domain walls for two system sizes showing that the distance on the Cayley tree $d$ is heavily bounded from above.

where the sum runs over all triangular plaquettes with upward orientation. Monte Carlo calculations of $\left\langle d_{\mathrm{av}}\right\rangle$ show that the average number of irreducible domain walls is indeed surprisingly small and definitely saturates at a finite value, see Fig. 9(a). On average, only

$$
\left\langle d_{\mathrm{av}}\right\rangle_{\infty}=1.9111(7)
$$

irreducible domain walls separate the domains at $k$ and $k^{\prime}(k)$ in the thermodynamic limit, when the distance between $k$ and $k^{\prime}(k)$ tends to infinity. This estimate was obtained using finitesize extrapolations according to Eq. (10), where in particular, for $L$ in the interval $[192,11340]$ one obtains the following fit parameters: $\lambda=1900(200), b=0.45(5), \chi^{2} /$ d.o.f $=1.4$. [In the same interval, a fit assuming purely algebraic decay of the correction, $g(x)=g_{1}+g_{2} L^{-g_{3}}$, has a $\chi^{2} /$ d.o.f $=300$ and a fit to a polynomial $p(x)=p_{1}+p_{2} / L+p_{3} / L^{2}+p_{4} / L^{3}$ has $\chi^{2} /$ d.o.f $=50$ highlighting that Eq. (10) provides a much better effective description.] Note that the value of the parameter $\lambda$ is somewhat larger than for the magnetization and chirality. The maximal number of irreducible domain walls ever observed during our simulations was 10, see Fig. 9(b).

Thus the simulation results presented in Fig. 9 clearly support the conclusions of Ref. 12 and confirm that spins 
$\mathbf{S}_{k}$ and $\mathbf{S}_{k^{\prime}(k)}$ belonging to the same sublattice indeed remain correlated, irrespective of the angle $\Phi$, even when the distance between them tends to infinity. This allows one to claim the existence of a long-range order in the Heisenberg model without making any reference to the pseudospin model.

Note that, in general, distribution functions such as the one shown in Fig. 9(b) contain useful physical information themselves. For instance, the chirality-chirality correlation function $\left\langle\chi_{k} \chi_{k^{\prime}(k)}\right\rangle$ is exactly given by summing over all the bins $d$ and counting the weight $p(d)$ with alternating signs

$$
\left\langle\chi_{k} \chi_{k^{\prime}(k)}\right\rangle=\sum_{d}(-1)^{d} p_{d},
$$

reflecting the sign change of the chirality upon crossing a domain wall. In our case, since $\langle|\chi|\rangle \approx 0.0005$, this correlation function is of the order of $10^{-7}$, which turns out to be difficult to resolve using Eq. (15) as this would require an accuracy of better than $10^{-8}$ of each histogram entry. It is, however, easy to check that Eq. (15) averages to a number very close to 0 with a large error bar that includes the true finite chirality.

The correlation function of the pseudospins at sites $k$ and $k^{\prime}(k)$ belonging to the same sublattice, $\left\langle\sigma_{k} \sigma_{k^{\prime}(k)}\right\rangle$, can be expressed in terms of $p_{d}$ as

$$
\begin{aligned}
\left\langle\sigma_{k} \sigma_{k^{\prime}(k)}\right\rangle= & p_{0}+\frac{1}{3} p_{1}-\frac{2}{6} p_{2}-\frac{4}{12} p_{3}+0 p_{4}+\frac{8}{48} p_{5}+\frac{8}{96} p_{6} \\
& -\frac{8}{192} p_{7}-\frac{24}{384} p_{8}-\frac{8}{768} p_{9}+\frac{40}{1536} p_{10}+\cdots,
\end{aligned}
$$

where we assumed that the distribution on the Cayley tree is isotropic, i.e., any possible irreducible sequence of $d$ domain walls occurs with equal probability. Under this assumption, the coefficient of $p_{d}$ in Eq. (16) is given by the average value of $\left\langle\sigma_{k} \sigma_{k^{\prime}(k)}\right\rangle$ at distance $d$ on the Cayley tree. In reality, the distribution on the Cayley tree is not exactly isotropic in finite systems. However, it can be expected to become isotropic in the thermodynamic limit. For $d=2$ and $L=5760$, we have checked that the substitution of the distribution function shown in Fig. 9(b) into Eq. (16) (based on the assumption that the distribution function is isotropic) leads to $\left\langle\sigma_{k} \sigma_{k^{\prime}(k)}\right\rangle=$ $0.085(3)$, which indeed is nicely consistent with the value of $m_{\alpha}^{2}\left(0.2925^{2} \simeq 0.0856\right)$ following from the more precise estimate of the sublattice magnetization [see Eq. (11)].

\section{Determination of the ground-state degeneracy}

To round up our numeric characterization of the groundstate of the noncoplanar phase of the classical bilinearbiquadratic Heisenberg model, we turn to the calculation of the residual entropy per site characterizing the ground-state degeneracy. When a system allows for independent local transformations that do not change its energy, the number of ground states $\Omega(L)$ can be expected to grow exponentially with the number of sites, that is, as

$$
\Omega(L)=c e^{\alpha L^{2}} .
$$

Accordingly, the entropy per site

$$
h(L)=\frac{1}{L^{2}} \ln \Omega(L)
$$

is expected to have a finite limit at $L \rightarrow \infty$,

$$
\lim _{L \rightarrow \infty} h(L)=\alpha>0
$$

Using a comparison with exactly solvable models Fendley et $a l{ }^{13}$ showed that the residual entropy $\alpha$ has to belong to the interval $0.3332<\alpha<0.3791$ and confirmed this by a numerical analysis, which gave an estimate $\alpha \approx 0.3661$. Our intention here is to improve the accuracy of this estimate on the basis of our efficient MC update and, in addition, to study whether the size dependence of the ground-state degeneracy also manifests some crossover phenomenon, in analogy to the magnetization and chirality.

Although the calculation of $\Omega$ is not directly possible within any of the above MC methods, one can combine these methods with the Wang-Landau scheme ${ }^{18}$ to obtain an estimate of the density of states $g(m)$ for all possible values of the total magnetization $m=\sum_{i} \sigma_{i}$. A basic version of this approach consists in using only the local MC updates in which the unnormalized density of states $\tilde{g}$ [which initially is assumed to be independent of $m, \tilde{g}(m) \equiv 1]$ is multiplied by a factor $f>1, \tilde{g}(m) \rightarrow f \tilde{g}(m)$, for any configuration of magnetization $m$ generated. Then, the only difference to the procedure applied above for the calculation of averages of different quantities consists in using as the acceptance probability for flipping a flippable spin $\sigma_{i}$ the dynamic quantity $\tilde{g}(m) / \tilde{g}\left(m-2 \sigma_{i}\right)$ instead of $1 / 2$. Conventionally, one iteration ends once all possible magnetizations have been seen (this can be checked by counting how often the simulation has observed magnetizations $\pm L^{2}$ and if it has traveled between those extremes) and if the histogram of the visited magnetizations is sufficiently flat. In the first iteration, one takes $f=e=2.718 \ldots$, but after each iteration, the modification factor $f$ is diminished $(f \rightarrow \sqrt{f}$ ) until $f$ becomes equal to 1 within some precision $\epsilon$ ( say $\left.\epsilon=10^{-7}\right)$.

In order to achieve the convergence at essentially larger system sizes than those which can be studied in the framework of this canonical Wang-Landau implementation, we modified the above basic strategy in the following (not necessarily unique) way. First, we employ the approach proposed by Zhou and Bhatt, ${ }^{19}$ which most notably relaxes the flat-histogram criterion by defining that a Wang-Landau iteration ends once each accessible microstate is sampled at least $1 / \ln f$ times. Second, we add occasional cluster moves to the sampling procedure applying the same acceptance rules as for the local Wang-Landau updates. The cluster updates are clearly useful as they can help to overcome barriers and to perform large jumps to phase space regions, which were not yet sufficiently sampled. Third — and probably most importantly-we parallelized the whole procedure on a multicore shared-memory architecture using the concurrent approach of Ref. 20 .

The density of states $\tilde{g}(m)$ thus obtained can be normalized using the condition that there are only two regular states in the pseudospin model having total magnetization $m= \pm L^{2}$, or $g\left(L^{2}\right)=g\left(-L^{2}\right)=1$. Hence an estimate for the number of ground-state configurations will be given by

$$
\Omega=\frac{1}{\tilde{g}\left(L^{2}\right)} \sum_{m=-L^{2}}^{m=L^{2}} \tilde{g}(m),
$$



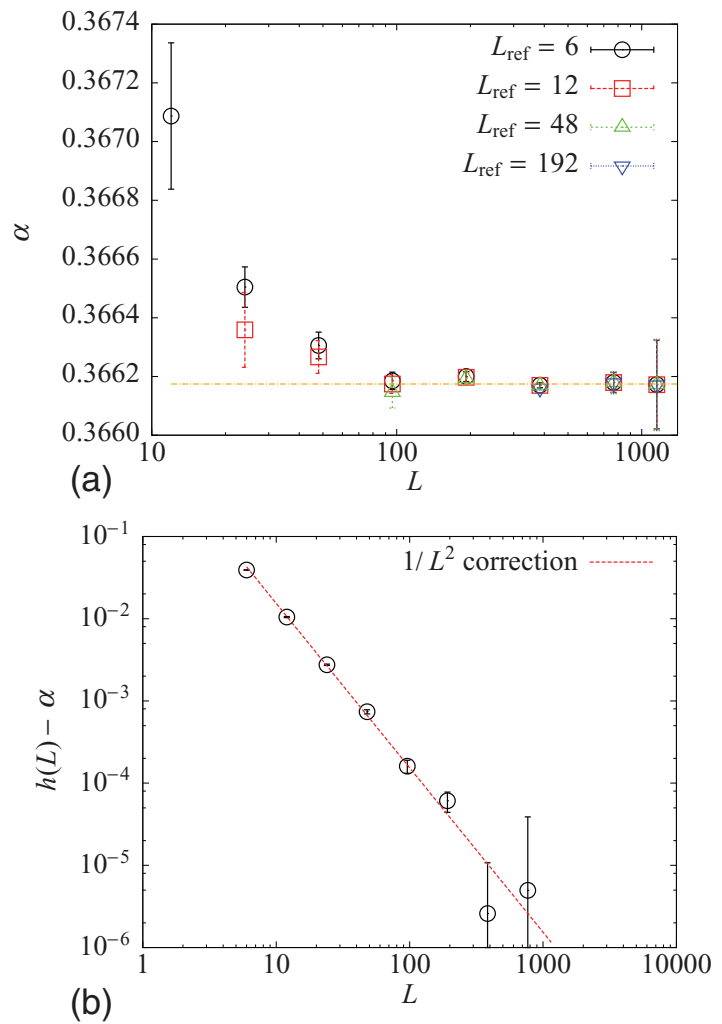

FIG. 10. (Color online) (a) Size dependence of the exponent $\alpha$ obtained from a pairwise comparison of ground-state degeneracies at sizes $L$ and $L_{\text {ref }}$. Curves using different fixed $L_{\text {ref }}$ collapse onto each other. The straight line is the result from the standard fit of $\Omega(L)$ to Eq. (17) for $L>24$. (b) The behavior of the size-dependent correction to entropy per site $h(L)$. Straight line corresponds to the $1 / L^{2}$ dependence.

where an uncertainty estimate is obtained by repeating the calculation 20 times with different pseudorandom numbers.

Using this strategy, we obtained rather accurate estimates for the number of ground-states $\Omega(L)$ on systems with linear size up to $L=1152$. A finite-size analysis of these numbers reveals that the dependence (17) is very nicely satisfied at all length scales, with almost no change in behavior between small and large length scales.

This is demonstrated in Fig. 10(a), which displays the size dependence of the exponent $\alpha$ calculated from the ground-state degeneracies $\Omega(L)$ and $\Omega\left(L_{\text {ref }}\right)$ at two different system sizes $L$ and $L_{\text {ref }}$ by using the relation

$$
\alpha=\frac{\ln [\Omega(L)]-\ln \left[\Omega\left(L_{\mathrm{ref}}\right)\right]}{L^{2}-L_{\mathrm{ref}}^{2}} .
$$

The reason to use two values of $\Omega$ at different $L$ is that this allows one to eliminate the unknown factor $c$ in Eq. (17). Curves using different choices for $L_{\text {ref }}$ converge and collapse onto each other for $L>100$, showing the validity of the scaling formula. The finite-size deviations at small $L$ are in the $1 \%$ region.

Moreover, the size dependence of the entropy per site $h(L)$, which we observe, is in perfect agreement with the predictions of the conformal analysis ${ }^{13,21,22}$ according to which at $L \rightarrow \infty$ the entropy per site $h(L)$ approaches its limit $\alpha$ as

$$
h(L) \approx \alpha+\frac{a}{L^{2}},
$$

consistent with Eq. (17). Our data adhere excellently to this scaling assumption and allow us to obtain an improved estimate for the residual entropy per site as

$$
\alpha=0.36618(1) \text {. }
$$

The data in Fig. 10(b) show that the finite-size corrections to this number follow the $1 / L^{2}$ dependence represented by the straight line.

Note that these data show no sign of a crossover effect for the scaling behavior of $\Omega(L)$ up to $L=1152$. An analogous analysis applied to the data on the size dependence of the chirality [presented in Fig. 8(a)] shows a visible tendency towards a change of regime-from an algebraic decay to a saturation-already at smaller sizes, whereas in the data for the size dependence of the magnetization [see Fig. 9(b)] the tendency to the change of regime is visible at even smaller scales without a special analysis. However, it has to be emphasized that in the case of $\Omega(L)$ a change of regime cannot be as drastic as for the magnetization or chirality and can consist only in a small change of the exponent $\alpha$ and therefore it may be much harder to observe its precursors at $L \lesssim 1000$.

The data presented in this section have been obtained by the method involving cluster updates and therefore corresponds to counting all allowed states of the pseudospin model. We also have verified that exclusion of the states not belonging to the Heisenberg sector does not lead to any noticeable change of the results. This means that the graphs presented in Fig. 10 and the value of the residual entropy given by Eq. (23) describe as well the properties of the original Heisenberg model.

\section{SUMMARY}

In conclusion, we have studied the zero-temperature properties of the noncoplanar phase of the classical bilinearbiquadratic Heisenberg model on the triangular lattice by means of extensive Monte Carlo simulations. Building on the characterization of the noncoplanar ground states in terms of zero-energy domain walls separating domains with different three-sublattice states, we have introduced an Ising pseudospin representation that allows for a particularly convenient description of the ground states without any reference to details such as the angle $\Phi$ between neighboring Heisenberg spins. The Ising representation gave rise to an elegant and efficient Monte Carlo procedure in which the space of allowed configurations is sampled by discrete pseudospin flips.

Both local and cluster Monte Carlo updates have been introduced, the latter being motivated by the need to simulate unusually large system sizes. Using a cluster Monte Carlo procedure, we have been able to study ground-state configurations in systems containing up to $1.3 \times 10^{8}$ spins. In addition to the basic characterization of the ground state degeneracy and of the domain-wall statistics, we have been able to unambiguously conclude on the nature of ordering in the noncoplanar phase at zero temperature. 
In terms of the pseudospin variables, evidence in favor of the presence of long-range magnetic order has been found already in systems of intermediate sizes, with linear size $L$ up to about 200. The existence of such an ordering had been predicted from analytical arguments in Ref. 12. Note that the earlier work of Fendley et al. ${ }^{13}$ was not conclusive in that respect and could not exclude a disordered state.

However, to reach a full understanding of the nature of ordering (in particular, to establish whether all three sublattices are ordered to an equal degree) turned out to be a highly nontrivial task. Only simulations on very large systems with $L \gtrsim 1000$ have given evidence that in the thermodynamic limit all three sublattices become equivalent, whereas below this length scale numerical simulations have not allowed the exclusion of a partially disordered state.

Although the existence of long-range order for Heisenberg spins follows already from the existence of the long-range order in the pseudospin model, ${ }^{12}$ we have also provided an additional proof for that directly for the Heisenberg spins by analyzing the distribution of the system in its order parameter space, which has the structure of an infinite Cayley tree of degree 3. It was shown that this distribution is highly localized and that two spin domains which are infinitely distant from each other are only separated on average by a finite number (roughly 2) of irreducible domain walls. This ensures the presence of long-range correlations for Heisenberg spins.

Importantly, the large-length simulations also provided evidence in favor of long-range chiral correlations in the pseudospin model, as well as for the noncoplanar ground states of the original Heisenberg model. In contrast to the ordering related to the orientation of Heisenberg spins (which at an arbitrarily low temperature is destroyed by continuous fluctuations-spin waves ${ }^{23,24}$ ), this order, which is related to a discrete degree of freedom, can be expected to survive at low enough temperatures, leading to a finite-temperature phase transition. The results of simulations of the bilinearbiquadratic Heisenberg model on the triangular lattice at finite temperatures have been reported in Ref. 11. However, the system sizes in this work never exceeded two hundred, and therefore the observation of ordering at low temperatures was rather a finite-size effect than the consequence of real chiral ordering which, according to our findings, is likely to exist only at much larger length scales.

We have also performed a high precision numerical calculation of the residual entropy of the model on the basis of a generalization of the Wang-Landau scheme. Our results agree with those of Ref. 13, but the accuracy has been increased by one order of magnitude. To our surprise, the size dependence of the ground-state degeneracy (which determines the value of the residual entropy) has not shown any sign of a crossover at $L \sim 1000$, as could have been expected from the existence of such a crossover in the behavior of other quantities (magnetization and chirality).

The most unusual feature of the model is the existence of a large length scale below which long-range order in terms of magnetization is combined with an algebraic decay of fluctuations and the complete disorder of the chirality. Only at sizes $L \gtrsim 1000$ does the behavior become more consistent with long-range order both in terms of the magnetization and chirality, and with an exponential leveling off of correlations.

The long-range order of the magnetization and of the chirality disappears in the special case of $\Phi=\arccos (-1 / 3)$, when the model becomes equivalent to the exactly solvable ${ }^{25}$ four-state antiferromagnetic Potts model on the triangular lattice, which is known to be critical. In terms of the loop representation this case corresponds to the presence of one more allowed vertex ${ }^{12,13}$ (a merging point of six domain walls) which destroys the possibility of constructing the pseudospin description of loop configurations. It seems plausible that even when they are allowed, such complex objects can be present only in low concentrations, because their presence strongly suppresses the possibilities of domain walls fluctuations. However, it eludes our understanding why the appearance of such rare defects shifts the system exactly into the critical point. In more familiar situations, the presence of rare topological defects induces the appearance of a finite correlation radius related to their concentration (like it happens in the antiferromagnetic Ising model on the triangular lattice at a finite temperature ${ }^{26}$ or in the conventional XY model above the Berezinkii-Kosterlitz-Thouless phase transition) and not its growth to infinity. In fact, the extreme largeness of the correlation radii we observed may precisely be related to the smallness of the concentration of the defects whose presence distinguishes the generic model from the special case $\Phi=\arccos (-1 / 3)$ situated exactly at criticality. In our opinion, this point deserves further investigation.

It should also be mentioned that the existence of a large length scale at which the properties of the system change qualitatively raises some questions about the analysis of Ref. 13, whose aim was to extract the central charge from the size dependence of the correction to the residual entropy. Our findings suggest that, since the numerical analysis of Ref. 13 relies on cylindrical systems with transverse sizes only up to $L=36$, it cannot be expected to provide reliable information on the behavior of the system in the thermodynamic limit.

\section{ACKNOWLEDGMENTS}

We are grateful for the support of the Swiss National Science Foundation, MaNEP, and the Hungarian OTKA Grant No. K106047 and are indebted to M. Hasenbusch and T. Vogel for useful discussions.
${ }^{1}$ B. Sutherland, Phys. Rev. B 12, 3795 (1975).

${ }^{2}$ L. A. Takhtajan, Phys. Lett. A 87, 479 (1982).

${ }^{3}$ H. M. Babujian, Phys. Lett. A 90, 479 (1982).

${ }^{4}$ I. Affleck, T. Kennedy, E. H. Lieb, and H. Tasaki, Phys. Rev. Lett. 59, 799 (1987).
${ }^{5}$ S. Nakatsuji, Y. Nambu, H. Tonomura, O. Sakai, S. Jonas, C. Broholm, H. Tsunetsugu, Y. Qiu, and Y. Maeno, Science 309, 1697 (2005).

${ }^{6}$ K. Harada and N. Kawashima, Phys. Rev. B 65, 052403 (2002). 
${ }^{7}$ H. Tsunetsugu and M. Arikawa, J. Phys. Soc. Jpn. 75, 083701 (2006).

${ }^{8}$ S. Bhattacharjee, V. B. Shenoy, and T. Senthil, Phys. Rev. B 74, 092406 (2006).

${ }^{9}$ A. Läuchli, F. Mila, and K. Penc, Phys. Rev. Lett. 97, 087205 (2006).

${ }^{10}$ T. A. Tóth, A. M. Läuchli, F. Mila, and K. Penc, Phys. Rev. B 85, 140403 (2012).

${ }^{11}$ H. Kawamura and A. Yamamoto, J. Phys. Soc. Jpn. 76, 073704 (2007).

${ }^{12}$ S. E. Korshunov, F. Mila, and K. Penc, Phys. Rev. B 85, 174420 (2012).

${ }^{13}$ P. Fendley, J. E. Moore, and C. Xu, Phys. Rev. E 75, 051120 (2007).

${ }^{14}$ U. Wolff, Phys. Rev. Lett. 62, 361 (1989).

${ }^{15}$ R. H. Swendsen and J.-S. Wang, Phys. Rev. Lett. 58, 86 (1987).
${ }^{16}$ J. Martín-Herrero, J. Phys. A 37, 9377 (2004).

${ }^{17}$ W. Press, S. Teukolsky, W. Vetterling, and B. Flannery, Numerical Recipes 3rd Edition: The Art of Scientific Computing (Cambridge University Press, Cambridge, 2007).

${ }^{18}$ F. Wang and D. P. Landau, Phys. Rev. Lett. 86, 2050 (2001).

${ }^{19}$ C. Zhou and R. N. Bhatt, Phys. Rev. E 72, 025701 (2005).

${ }^{20}$ L. Zhan, Comput. Phys. Commun. 179, 339 (2008).

${ }^{21}$ H. W. J. Blöte, J. L. Cardy, and M. P. Nightingale, Phys. Rev. Lett. 56, 742 (1986).

${ }^{22}$ I. Affleck, Phys. Rev. Lett. 56, 746 (1986).

${ }^{23}$ A. M. Polyakov, Phys. Lett. B 59, 79 (1975).

${ }^{24}$ P. Azaria, B. Delamotte, T. Jolicoeur, and D. Mouhanna, Phys. Rev. B 45, 12612 (1992).

${ }^{25}$ R. J. Baxter, J. Math. Phys. 11, 784 (1970).

${ }^{26}$ B. Nienhuis, H. J. Hilhorst, and H. W. J. Blote, J. Phys. A 17, 3559 (1984). 